\title{
A 1 V supercapacitor device with nanostructured graphene oxide/polyaniline composite materials
}

\author{
DEEPAK KUMAR, ANJAN BANERJEE*, SATISH PATIL and ASHOK K SHUKLA \\ Solid State and Structural Chemistry Unit, Indian Institute of Science, Bangalore 560012, India
}

MS received 17 April 2015; accepted 29 June 2015

\begin{abstract}
Polyaniline and graphene oxide composite on activated carbon cum reduced graphene oxide-supported supercapacitor electrodes are fabricated and electrochemically characterized in a three-electrode cell assembly. Attractive supercapacitor performance, namely high-power capability and cycling stability for graphene oxide/polyaniline composite, is observed owing to the layered and porous-polymeric-structured electrodes. Based on the materials characterization data in a three-electrode cell assembly, $1 \mathrm{~V}$ supercapacitor devices are developed and performance tested. A comparative study has also been conducted for polyaniline and graphene oxide/polyaniline composite-based $1 \mathrm{~V}$ supercapacitors for comprehending the synergic effect of graphene oxide and polyaniline. Graphene oxide/polyaniline composite-based capacitor that exhibits about $100 \mathrm{~F} \mathrm{~g}^{-1}$ specific capacitance with faradaic efficiency in excess of $90 \%$ has its energy and power density values of $14 \mathrm{Wh} \mathrm{kg}^{-1}$ and $72 \mathrm{~kW} \mathrm{~kg}^{-1}$, respectively. Cycle-life data for over 1000 cycles reflect $10 \%$ capacitance degradation for graphene oxide/polyaniline composite supercapacitor.
\end{abstract}

Keywords. Supercapacitor; pseudocapacitance; polyaniline; graphene oxide; cycling stability.

\section{Introduction}

Carbon-based supercapacitors, ${ }^{1-3}$ both with aqueous and non-aqueous electrolytes, have dominated the R\&D activity on electrochemical capacitors during the last few decades. Ironically, however, low energy density of such supercapacitors has been the limiting factor for wider exploitation. To overcome the aforesaid limitation, researchers are introducing fast surface faradaic charging with the electrical-double-layer capacitors. In the arena of pseudocapacitors, ${ }^{3,4}$ transition metal oxides and ceratin nitrides have looked attractive due to their long cycling stability. On the other hand, in recent years, conducting polymers are being explored as possible materials for supercapacitors owing to their flexibility, ${ }^{5}$ low $\operatorname{cost}^{6}$ and high conductivity in doped states ${ }^{7,8}$ with little environmental hazards. ${ }^{9}$ Besides, capacitance values higher than with electrical-double-layer capacitors are possible to achieve due to fast pseudo-faradaic charge-transfer. However, a drawback of conducting polymer-based supercapacitors is their poor cycling capability due to structural instability during charge/discharge processes. The large volumetric swelling and shrinking during doping and dedoping lead to poor lifetime. ${ }^{10}$ Due to the low internal resistance in doped state of conducting polymers, such as polyaniline and polypyrrole, the power capabilities for the supercapacitors are high but their poor cycling stability continues to be a limiting factor.

\footnotetext{
*Author for correspondence (anjansc.ac@gmail.com)
}

In the literature, efforts are being expended to mitigate the aforesaid problems by forming composites between the carbon nanomaterials and conducting polymers. ${ }^{11}$ The composite materials have improved capacitance values and good cycling stability, as conducting polymers enhance the capacitance of carbon nanomaterials by introducing voltage-dependent pseudocapacitance, while carbon nanomaterials, such as multiwalled carbon nanotubes, graphene, graphene oxide (GO) and reduced graphene oxide ( $\mathrm{rGO}$ ), help to increase their mechanical stability and cycle-life. In this context, GO and conducting polymer composites have been extensively investigated due to their exceptional mechanical and electrical properties. ${ }^{12}$ The availability of functional groups on GO, such as hydroxyl, epoxide, carbonyl and carboxyl groups on its basal planes and edges, can be utilized for chemical functionalization of conducting polymers. ${ }^{13}$ Typically, its good dispersion stability in aqueous and other common organic solvents provides a facile route to prepare GO/polymer composites, which are considered to be attractive attributes for these materials in improving the performance of supercapacitors.

Various methods are reported to improve the cycle-life of conducting polymer-based supercapacitors. For example, Zhu et $\mathrm{l}^{14}$ showed that polyaniline/sulphonated multi-walled carbon nanotubes composite loses $\sim 10 \%$ of its initial capacitance after 1000 cycles. Zhang and Zhao ${ }^{15}$ reported that polyaniline and polypyrrole deposited on rGO substrate can reach capacitance degradation of $\sim 18$ and $\sim 19 \%$, respectively, after 1000 cycles. Fu et al reported that carbon nanotube embedded in polypyrrole nanowire electrode loses 
about $15 \%$ of capacitance after testing for 1000 cycles at $1 \mathrm{~A} \mathrm{~g}^{-1}$ current density. ${ }^{16}$ Wang et $a l^{17}$ demonstrated that supercapacitor electrode with polyaniline deposited on coral-like monolithic carbon achieves capacitance degradation of about $22 \%$ after 1000 cycles at a scan rate of $100 \mathrm{mV} \mathrm{s}^{-1}$. Cycling stability of polymer electrodes is also studied beyond 1000 cycles, for example, Liu et $\mathrm{l}^{18}$ demonstrated that the capacitance degradations for carbonaceous shell-coated polyaniline and polypyrrole electrodes are $\sim 5$ and $\sim 15 \%$, respectively, after 10000 cycles at a scan rate of $100 \mathrm{mV} \mathrm{s}^{-1}$. Kim et al, ${ }^{19}$ in one of his studies, synthesized polyaniline/reduced graphene nanosheet composites by polymerization through chemical oxidation while changing the weight ratio of aniline monomers and characterized the capacitance behavior of the materials using a three-electrode system. It is noteworthy that most of the reports in the literature on the conducting polymerbased supercapacitors are single-electrode characterization studies and their performance as a device is lacking.

To exploit the aforesaid attributes of nanomaterial/polymer composites, in this study, we have attempted to improve the cycling stability of polyaniline by in situ electropolymerization of aniline in the presence of GO templates. The effect of $\mathrm{GO}$ on the electrochemical performance of supercapacitors was studied in a three-electrode as well as in a two-electrode electrochemical cell assembly. The rated specific-capacitance values for polyaniline-based $1 \mathrm{~V}$ supercapacitor devices are 100 and $80 \mathrm{~F} \mathrm{~g}^{-1}$ with the capacitance degradation of about 10 and $30 \%$ after 1000 cycles with and without GO, respectively. The power density is also higher for $\mathrm{GO} /$ polyaniline composite-based supercapacitor due to its shorter response time.

\section{Experimental}

\subsection{Synthesis and characterization of $G O$ and $r G O$}

GO and rGO were synthesized from graphite flakes by modified Hummer's method and hydrazine hydrate, respectively,

Table 1. Active material compositions for three-electrode cell assembly.

\begin{tabular}{ll}
$\begin{array}{l}\text { Working electrode } \\
\text { designation }\end{array}$ & Active material composition \\
\hline WE A & $\begin{array}{c}85 \% \text { Activated carbon }+10 \% \text { rGO } \\
+5 \% \text { PVDF binder }\end{array}$ \\
WE B & PANI coating on top of WE A \\
WE C & GO/PANI coating on top of WE A \\
\hline
\end{tabular}

as reported elsewhere. ${ }^{20,21}$ The detailed synthesis is described in the supplementary information. Synthesized GO and rGO were characterized by Fourier transform infrared (FT-IR) spectroscopy (supplementary figure S1) and Raman spectroscopy (supplementary figure S2). Characteristic peaks in FT-IR spectra and bands in Raman spectra support the formation of GO from graphite flakes and further modification to rGO was analysed by chemical method.

\subsection{Fabrication of working electrodes}

Activated carbon-coated graphite working electrodes were fabricated using dimethyl formamide as a solvent and $5 \mathrm{wt} \%$ poly(vinylidene fluoride) (PVDF) as a binder. Subsequently, the mixture was blended with $85 \mathrm{wt} . \%$ activated carbon and 10 wt. $\%$ rGO to form an ink. Resulting carbon ink was applied onto the graphite substrate followed by drying in an air-oven at $80^{\circ} \mathrm{C}$ for $6 \mathrm{~h}$.

\subsection{Electropolymerization of polyaniline and GO/polyaniline composite}

rGO-embedded activated carbon working electrodes were amperometrically coated with a thin layer of polyaniline (PANI) in a three-electrode cell assembly using $0.5 \mathrm{M}$ sulphuric acid and $0.2 \mathrm{M}$ aniline solution under nitrogen purging at $0.75 \mathrm{~V}$ vs. $\mathrm{Ag} / \mathrm{AgCl}$ reference electrode. ${ }^{22}$ The optimized duration for PANI electrodeposition was $5 \mathrm{~min}$. Electrodeposition of GO and PANI composite was carried out at the same potential as mentioned above. The water suspension of GO was prepared by $1 \mathrm{~h}$ ultrasonic agitation, while the optimized concentration of GO was $60 \mathrm{mg} \mathrm{l}^{-1}$. Subsequently, aniline and sulphuric acid were added to GO suspension. Resulting solution was used for electrodeposition of GO/PANI composite under nitrogen purging; optimized period for electrodeposition was $3 \mathrm{~min}$. Amperometric profiles for electrodeposition of PANI and GO/PANI composite are presented in supplementary figure S3. Resulting PANI and GO/PANI composite were characterized by FT-IR spectroscopy, and corresponding data are shown in supplementary figure S4.

\subsection{Configuration of electrochemical cells}

Electrochemical characterization of electrode-active materials was carried out by assembling a three-electrode cell in a Plexiglass container, in which active materials were applied on the working electrode $\left(1.5 \times 1.5 \mathrm{~cm}^{2}\right.$ geometric area). A graphite sheet with a similar geometric area and an $\mathrm{Ag} / \mathrm{AgCl}$

Table 2. Electrode combinations for $1 \mathrm{~V}$ supercapacitor devices in two-electrode cell assembly.

Positive electrode

Supercapacitor A

Supercapacitor B
(85\% Activated carbon $+10 \%$ rGO $+5 \%$ PVDF binder $)$ + PANI coating (WE B)

( $85 \%$ Activated carbon $+10 \% \mathrm{rGO}+5 \%$ PVDF binder $)$ + GO/PANI coating (WE C)
Negative electrode

$85 \%$ Activated carbon $+10 \%$ rGO $+5 \%$ PVDF binder (WE A)

$85 \%$ Activated carbon $+10 \%$ rGO $+5 \%$ PVDF binder (WE A) 
electrode were used as the counter and the reference electrodes, respectively. Three different kind of working electrodes were made, namely $85 \%$ activated carbon $+10 \%$ rGO $+5 \%$ PVDF (WE A), $85 \%$ activated carbon $+10 \%$ rGO $+5 \%$ PVDF + PANI (WE B) and $85 \%$ activated carbon + $10 \%$ rGO + 5\% PVDF + GO/PANI (WE C), which are summerized in table 1. Optimum concentration of sulphuric acid electrolyte was chosen as $2 \mathrm{M}$. Detailed optimization data are presented in supplementary figure S5.

\subsection{Evaluating the supercapacitor performance}

Two-electrode cell assemblies were designed for $1 \mathrm{~V}$ supercapacitor devices. The electrode combinations of supercapacitor devices are presented in table 2. Geometric areas of electrodes were $3 \times 3.5 \mathrm{~cm}^{2}$, and the two electrodes were separated by $0.5 \mathrm{~mm}$ polyethylene mesh. Sulphuric acid ( $2 \mathrm{M})$ was used as electrolyte. $1 \mathrm{~V}$ supercapacitor devices thus assembled were performance tested by various $\mathrm{dc}$ and ac electrochemical methods using Autolab Potentiostat/ Galvanostat-Model 30 at room temperature $\left(\sim 25^{\circ} \mathrm{C}\right)$.

\section{Results and discussion}

\subsection{Electrode materials characterization}

Figure 1a-c shows surface morphologies for various working electrodes, namely WE A, WE B and WE C, respectively. However, figure 1a shows the activated carbon and rGO composite-based active material structure, wherein the respective particle size values for activated carbon and rGO range between 5-10 and 2-5 $\mu \mathrm{m}$. A scanning electron micrograph of rGO is also shown in the inset to figure 1a. Smallersized and highly conducting rGO improves the inter-particle contacts among comparatively larger-sized and less conducting activated carbon particles and helps reducing the internal resistance. Besides rGO contributes to the voltage-dependent pseudocapacitive component along with electrical-doublelayer capacitance, and, consequently, resulting capacitance value is increased substantially. A quantitative data on the effect of rGO in reducing internal resistance that concomitantly improves capacitance is presented in supplementary figures S6 and S7. Figure 1b shows PANI nano-fibres with about $100 \mathrm{~nm}$ diameter. Porous, fibrous and random polymer matrix covers the basement of activated carbon and rGO composite layer, and enhances the effective surface area of the electrode. Effective capacitance increases due to the increase in electrical-double-layer capacitance owing to higher surface area and pseudocapacitance for fast electron transfer among the different redox states of PANI. Figure 1c shows porous, fibrous and layered polymer matrix with about $100 \mathrm{~nm}$ fibre thickness, which also covers the carbon layer. The layered structure of the polymer matrix is clearly seen in high magnification image in the inset to figure 1c. Layered structure of GO particles acts as template for layered polymeric matrix in electropolymerization. Non-covalent H-bonding interactions between oxygen containing functionalities of

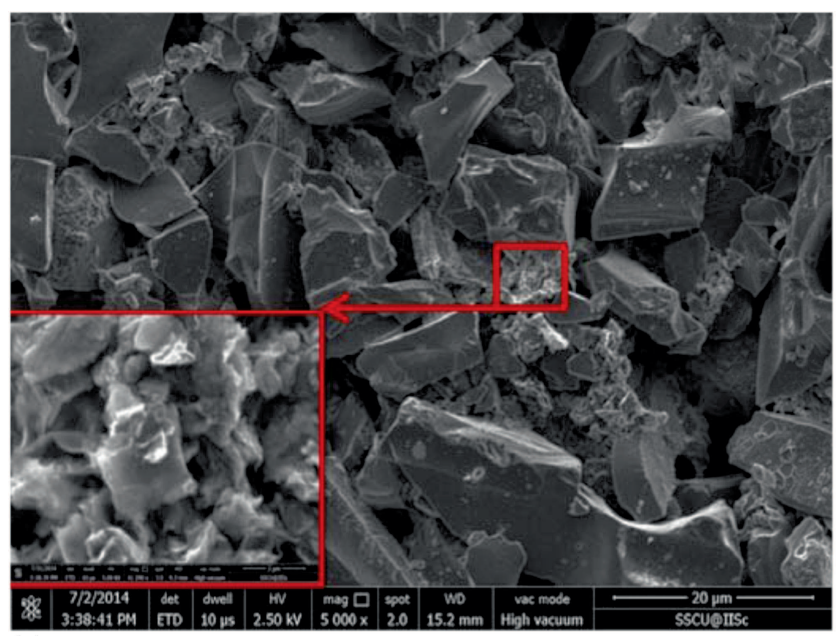

(a)

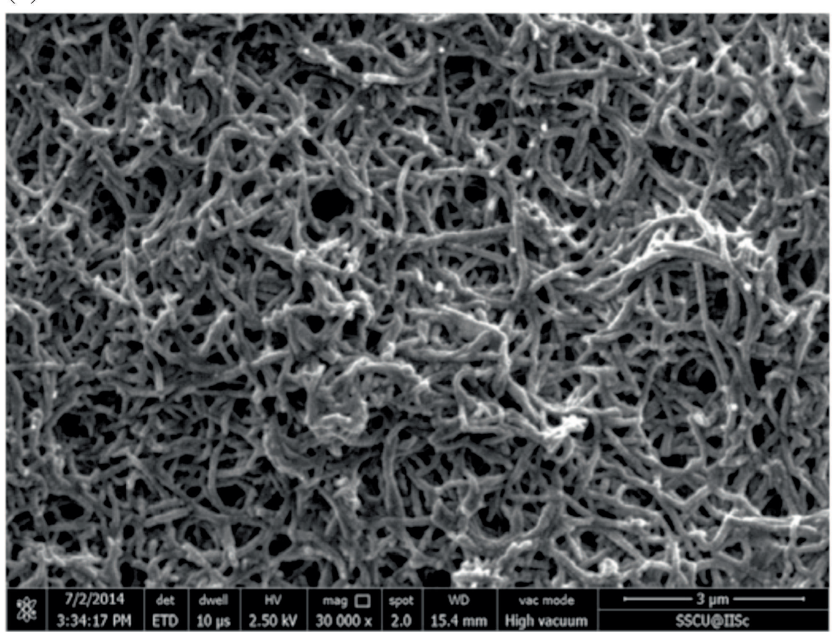

(b)

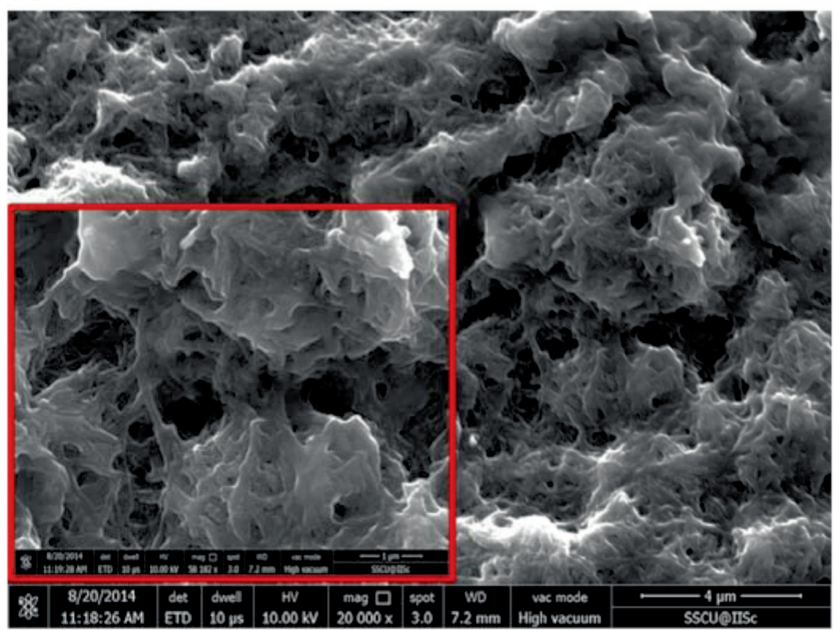

(c)

Figure 1. Scanning electron surface micrograph for (a) WE A (inset: structural morphology of rGO), (b) WE B and (c) WE C (inset: layered polymeric matrix at higher magnification).

GO, such as hydroxyl, epoxide, carbonyl and carboxyl groups on its basal planes and edges, and amine functionalities of aniline monomer and polymer direct the nucleation/growth of polymeric matrix. ${ }^{11}$ Consequently, GO particles are 
covered with polymer layers bringing about improved mechanical stability of polymer matrix. To further investigate the structure of GO/PANI composite, transmission electron microscopic (TEM) studies were carried out. Figure 2a shows TEM image of GO/PANI, while the dark/bright contrast on the polymer rod indicates the core-shell structure. Inset to figure $2 \mathrm{a}$ shows selected area electron diffraction (SAED) pattern, which reflects that the composite material is not highly crystalline. In the high-resolution transmission electron microscopy (HR-TEM) image, shown as figure $2 \mathrm{~b}$, good lattice fringes are visible in darker region compared to the lighter region, which justifies the coating of only a meagre amount of crystalline PANI on top of more crystalline GO supports. Furthermore, HR-TEM image shows inter-planer spacing of GO, namely 0.21 and $0.34 \mathrm{~nm}$ for (100) and (311) lattice planes at $2 \theta=42.7^{\circ}$ and $25.7^{\circ}$, respectively, which synchronize well with the PXRD pattern of GO. ${ }^{23,24}$ This study strongly validates the composite core-shell structure for GO and PANI.

\subsection{Cyclic voltammetric study}

Figure 3a-c depicts cyclic voltammograms for various working electrodes, namely WE $\mathrm{A}, \mathrm{WE} \mathrm{B}$ and $\mathrm{WE} \mathrm{C}$, respectively, in a three-electrode cell assembly between -0.2 and $0.8 \mathrm{~V}$ working voltage windows with respect to the reference
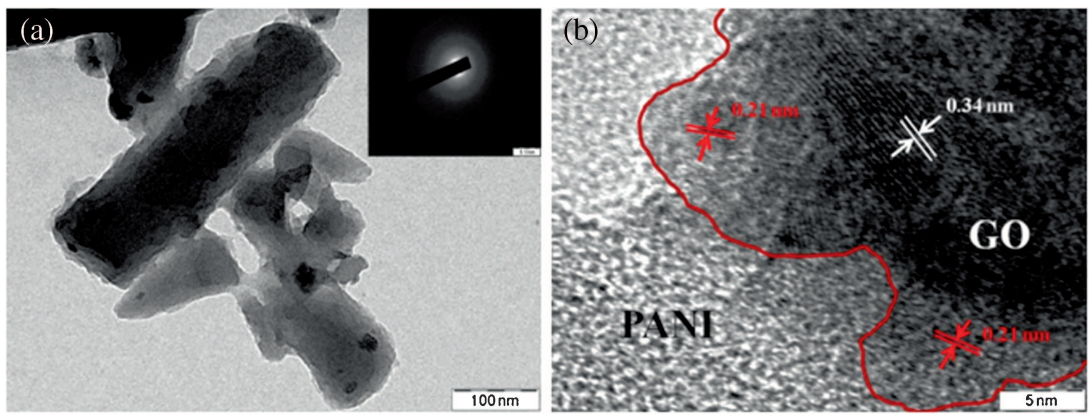

Figure 2. (a) TEM and (b) HR-TEM images for GO/PANI composite material. Inset of a shows the SAED pattern of composite.
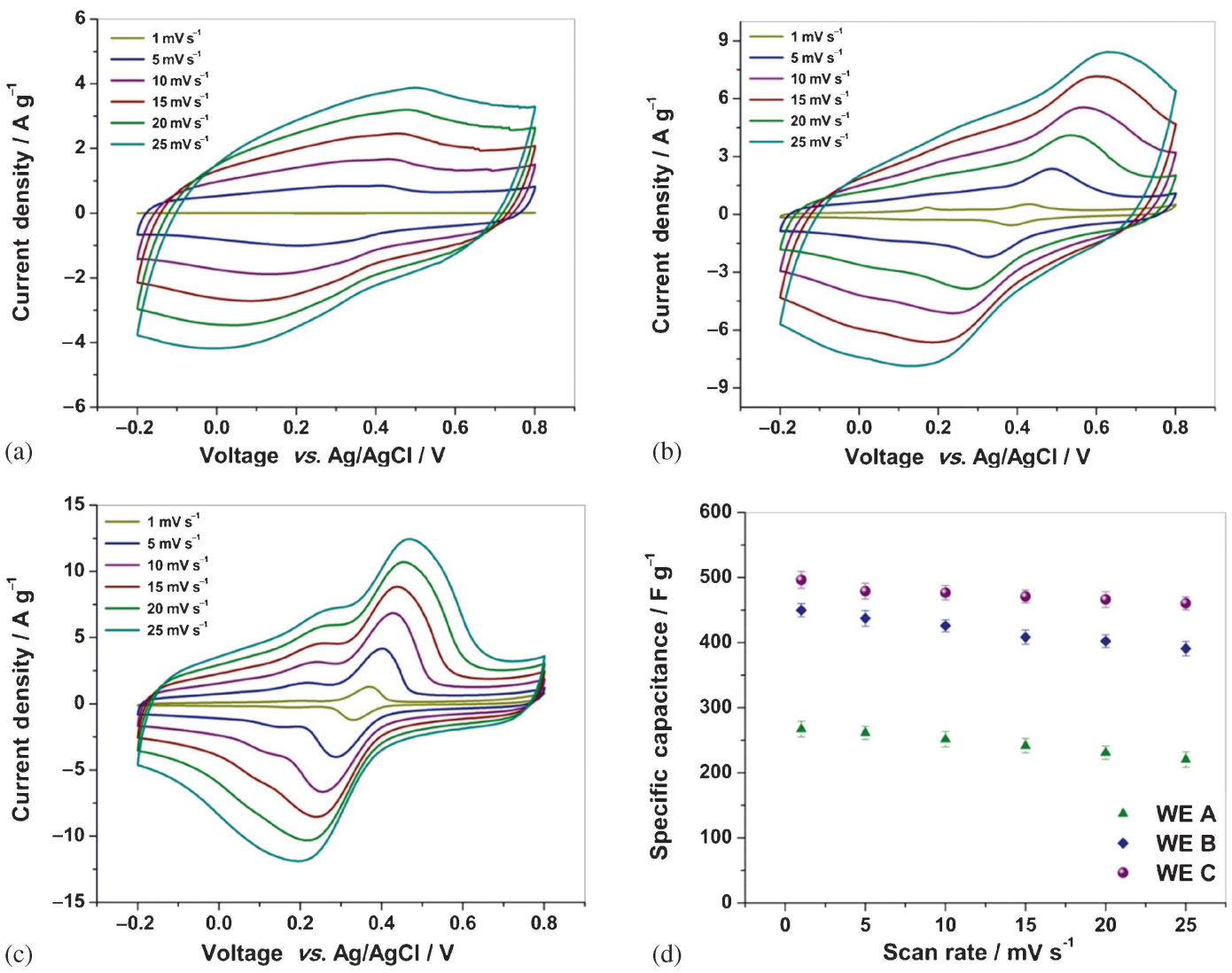

Figure 3. Cyclic voltammograms at different scan rates for (a) WE A, (b) WE B and (c) WE C. (d) Scan rate vs. specific capacitance profiles for WE A, WE B and WE C. 
electrode at varying scan rates. The specific-capacitance values are calculated from the cyclic voltammograms using equation (1), and respective data are presented in figure $3 \mathrm{~d}$.

Specific capacitance $=\frac{\text { Area under the cyclic voltammogram }}{v \times \Delta V \times m}$

In equation (1), $v, \Delta V$ and $m$ are the scan rate, working voltage window and mass of active materials, respectively. High specific capacitance values are achieved for WE C, owing to the synergistic effect of GO/PANI composite. WE B shows higher capacitance than WE A for the larger pseudocapacitance component of PANI. Due to the presence of rGO in WE A, certain amount of facilitated pseudocapacitance is observed in addition to electrical-double-layer capacitance, as shown in figure $3 \mathrm{a}$, which improves the resulting capacitance of carbon-based layers in the working electrodes. It is seen from figure $3 \mathrm{~b}$ and $\mathrm{c}$ that the pseudocapacitance overpotential is reduced and the shifts in overpotentials at fast scan rates are lowered in the presence of GO. The diffusion path-length for ions through the layered porous matrix in WE $C$ is shorter compared with random porous matrix in $\mathrm{WE} \mathrm{B}$, and therefore the resistive component arising from mass-transport limitation is lesser for WE C than WE B.
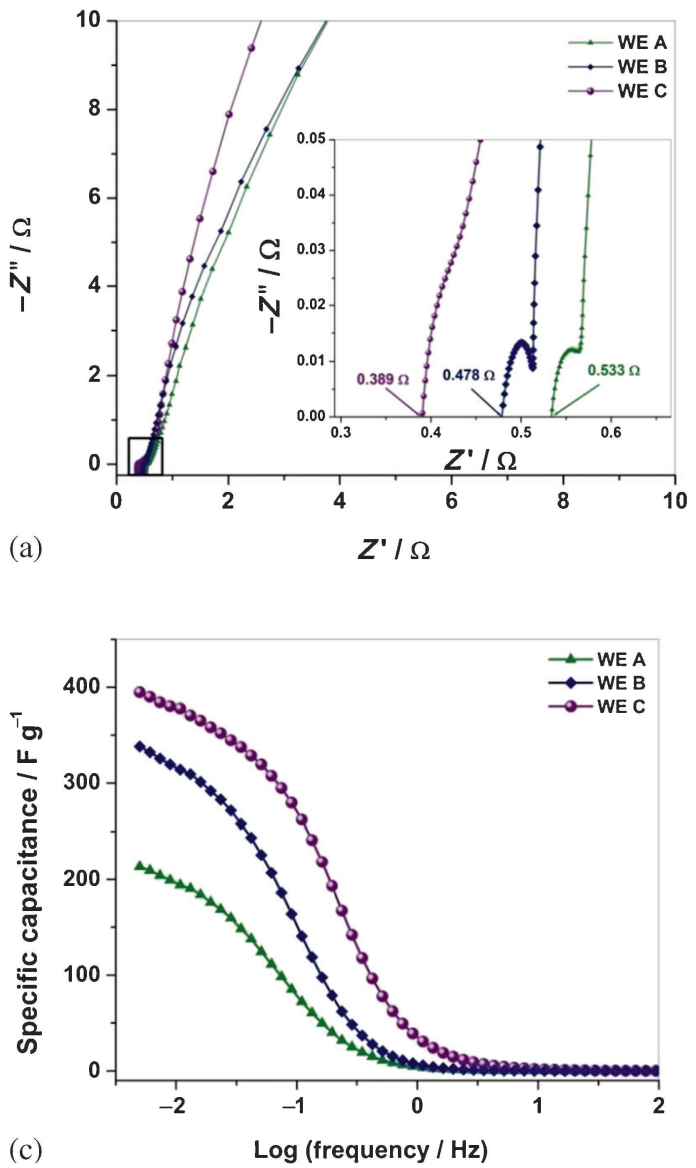

\subsection{Impedance analysis}

Frequency-response analysis through electrochemical impedance spectroscopy is performed for WE A, WE B and WE C. Figure 4a shows Nyquist plots for aforementioned working electrodes. X-intercept in Nyquist plots at higher frequency is considered as internal resistance, ${ }^{25}$ and the estimated internal-resistance values are: $0.533,0.478$ and $0.389 \Omega$ for WE A, WE B and WE C, respectively. Conducting polymer structures improve the internal resistance for $\mathrm{WE} \mathrm{B}$ and WE $\mathrm{C}$ in relation to WE $\mathrm{A}$. The layered-polymer matrix in WE $\mathrm{C}$ further reduces its internal-resistance value in relation to $\mathrm{WE} \mathrm{B}$. Also, the $\pi-\pi$ stacking interaction between GO sheet and PANI enhances the electron-transfer efficiency for the composite material, which reduces the internal resistance. ${ }^{26}$ The response-time data are calculated from Bode plots (frequency vs. phase) ${ }^{25}$ these data follow the same trend as internal-resistance values. The shorter ionic-diffusion pathway through the layered porous matrix is responsible for a shorter response-time for $\mathrm{WE} \mathrm{C}$ in relation to $\mathrm{WE} \mathrm{B}$. The higher response-time for $\mathrm{WE} \mathrm{A}$ is due to higher internal-resistance value. Figure $4 \mathrm{~b}$ shows the Bode plots for different working electrodes. Response-time values are: 1.7, 1.2 and $0.6 \mathrm{~s}$ for $\mathrm{WE} \mathrm{A,} \mathrm{WE} \mathrm{B} \mathrm{and} \mathrm{WE} \mathrm{C,}$
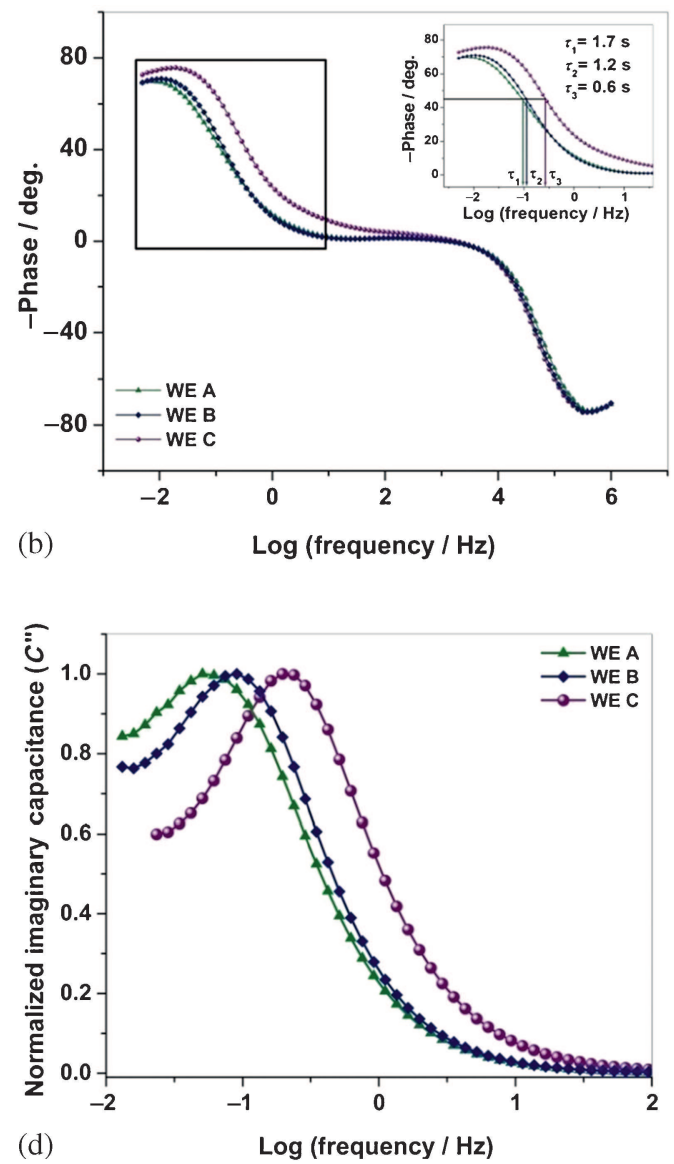

Figure 4. Frequency-response analysis by electrochemical impedance spectroscopy for different working electrodes: (a) Nyquist plots, (b) Bode plots, (c) real capacitance vs. frequency plots and (d) imaginary capacitance $v s$. frequency plots. 
respectively, as derived from the frequency values $(f)$ at phase angle $-45^{\circ}$ using equation (2). ${ }^{27}$

$$
\text { Response time }=\frac{1}{2 \times \pi \times f}
$$

The frequency dependent capacitance $(C)$ is a combination of real $\left(C^{\prime}\right)$ and imaginary $\left(C^{\prime \prime}\right)$ parts according to equation (3). The imaginary part of capacitance signifies the irreversible energy dissipation and corresponds to the relaxation process during ion transport. ${ }^{28} \mathrm{~A}$ measure has been taken to separate out these two parts from impedance spectroscopy using equations (4) and (5), ${ }^{28}$

$$
\begin{aligned}
& C(f)=C^{\prime}(f)+i C^{\prime \prime}(f), \text { where } i=\sqrt{ }-1 \\
& C^{\prime}(f)=\frac{-Z^{\prime \prime}(f)}{2 \times \pi \times f \times|Z(f)|^{2}} \\
& C^{\prime \prime}(f)=\frac{Z^{\prime}(f)}{2 \times \pi \times f \times|Z(f)|^{2}}
\end{aligned}
$$

where $Z^{\prime}, Z^{\prime \prime}$ and $|Z|$ represent the real part (resistance), imaginary part (reactance) and modulus of total impedance, respectively, and $Z=Z^{\prime \prime}+i Z^{\prime \prime}$. Figure $4 \mathrm{c}$ shows the frequency response for real part of capacitance, and it is observed that from higher to lower frequency region the capacitance values increase from about zero to near saturation. The capacitance values at any particular frequencies decrease from WE C to WE B to WE A, and, as a result, the power capability is highest for WE $\mathrm{C}$ and lowest for WE A. The higher power capability is achieved for WE C due to the lower internal resistance and shorter responsetime values. Figure $4 \mathrm{~d}$ shows the imaginary part of capacitance $v s$. frequency profiles for WE A, WE B and WE C with maximum at relaxation or breaking frequencies; ${ }^{25,28}$ the maximum value indicates the maximum energy dissipation from the system. ${ }^{28}$ The relaxation or breaking frequencies are the transition points from resistive (high frequency) to capacitive (low frequency) characteristics in systems, and the reciprocals of these frequencies (angular-frequency forms) match with response-time values. Response-time data from figure $4 \mathrm{~b}$ and $\mathrm{d}$ match each other.

\subsection{Galvanostatic charge/discharge data}

Figure 5a-c exhibits galvanostatic charge/discharge profiles at varying current densities within working voltage window for WE A, WE B and WE C. Figure 5d shows the calculated specific-capacitance values from the galvanostatic discharge plots at varying current densities according to the
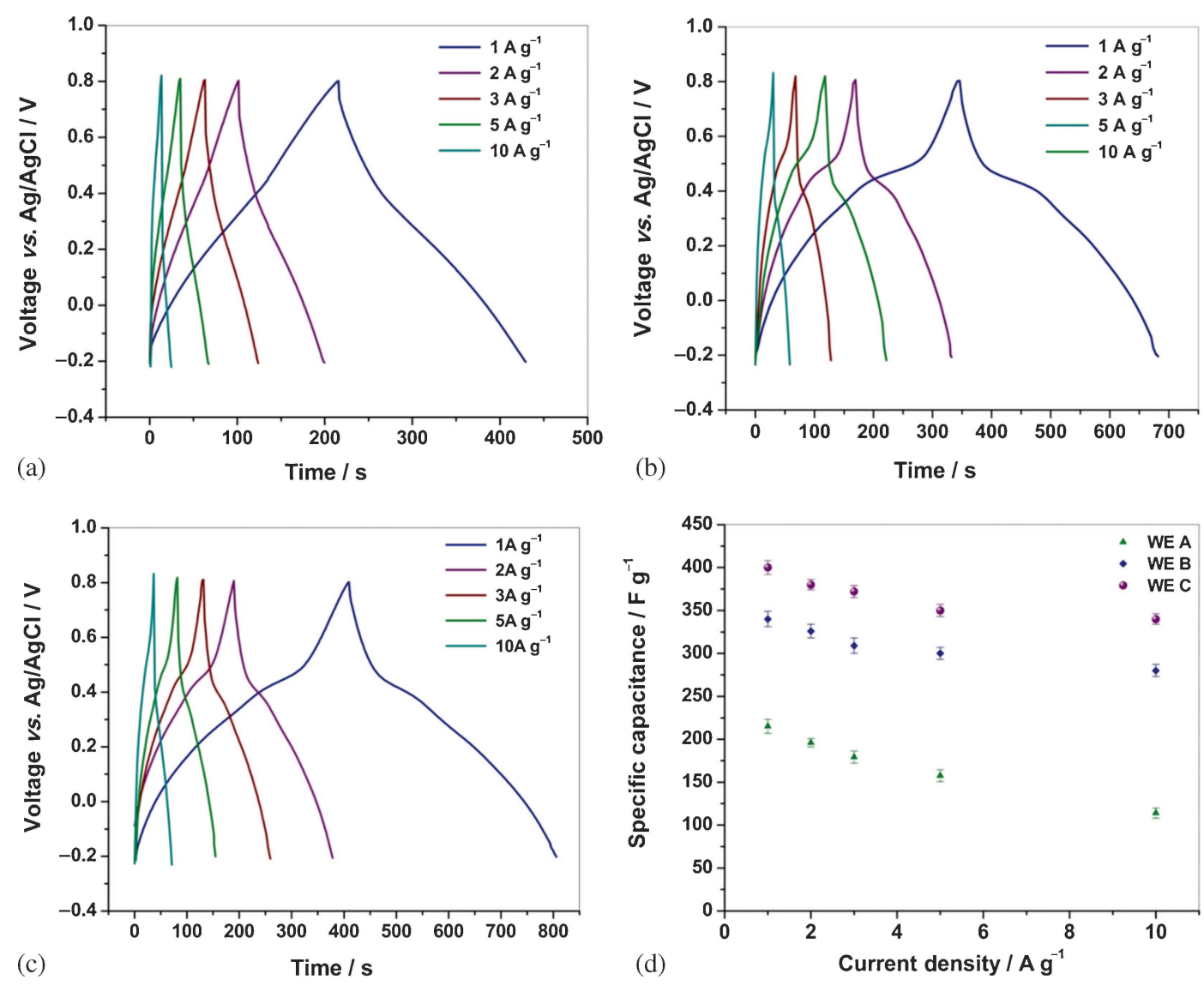

Figure 5. Galvanostatic charge/discharge profiles at different current densities for (a) WE A, (b) WE B and (c) WE C. (d) Current density vs. specific capacitance profiles for WE A, WE B and WE C. 


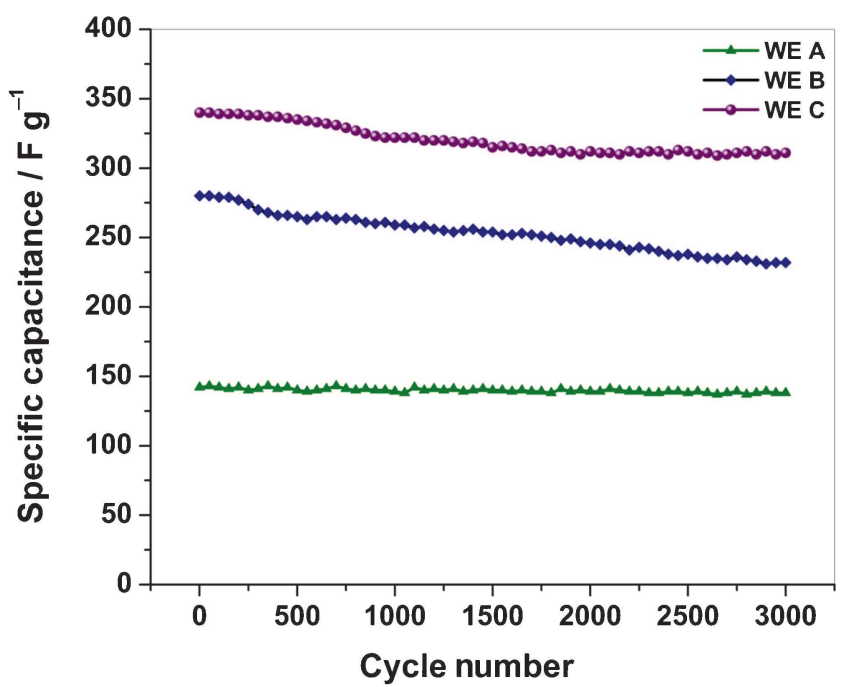

Figure 6. Cycle-life data for working electrodes WE A, WE B and $\mathrm{WE} \mathrm{C}$ at $10 \mathrm{~A} \mathrm{~g}^{-1}$ current density.

equation (6), where $I$ and $t$ represent the discharge current and time, respectively.

$$
\text { Specific capacitance }=\frac{I \times t}{\Delta V \times m}
$$

Faradaic efficiency values are calculated from galvanostatic charge/discharge profiles by using the ratio of charge (in coulomb) output and input, and it is observed that the faradaic efficiency values are $>95 \%$ at all current densities as shown in figure 4. It is noteworthy that due to the non-uniform current distribution in the porous matrix, the specific-capacitance values decrease at higher current densities, ${ }^{29}$ which is in accordance with the data obtained from cyclic voltammetry at different scan rates.

\subsection{Cycle-life data}

Cycle-life data for different working electrodes are obtained through galvanostatic charge/discharge at $10 \mathrm{~A} \mathrm{~g}^{-1}$ current density, and the data are shown in figure 6 . The capacitance values decrease by 3,17 and $8.5 \%$ after 3000 cycles for WE A, WE B and WE C, respectively. Scanning electron micrographs on the surfaces of WE A, WE B and WE C are recorded after 3000 cycling, and are presented in figure $7 \mathrm{a}-\mathrm{c}$. Figure $7 \mathrm{a}$ shows unaffected electrode surface morphology after 3000 cycles, which is responsible for better cycle-life of WE A. From figure 7b, it is clearly evident that the polymeric structures are disrupted, and the basement carbon layer is visible. By contrast, the polymeric structures are quite stable in figure $7 \mathrm{c}$ due to the increased mechanical stability of polymeric matrix in the presence of GO templates. The trend for capacitance degradation in WE B and WE C is reflected by the structural stability of polymer matrixes.

\subsection{Electrochemical performance data for $1 \mathrm{~V}$ supercapacitors}

In the light of the foregoing, $1 \mathrm{~V}$ supercapacitor devices, namely supercapacitor A and supercapacitor B (table 2) are

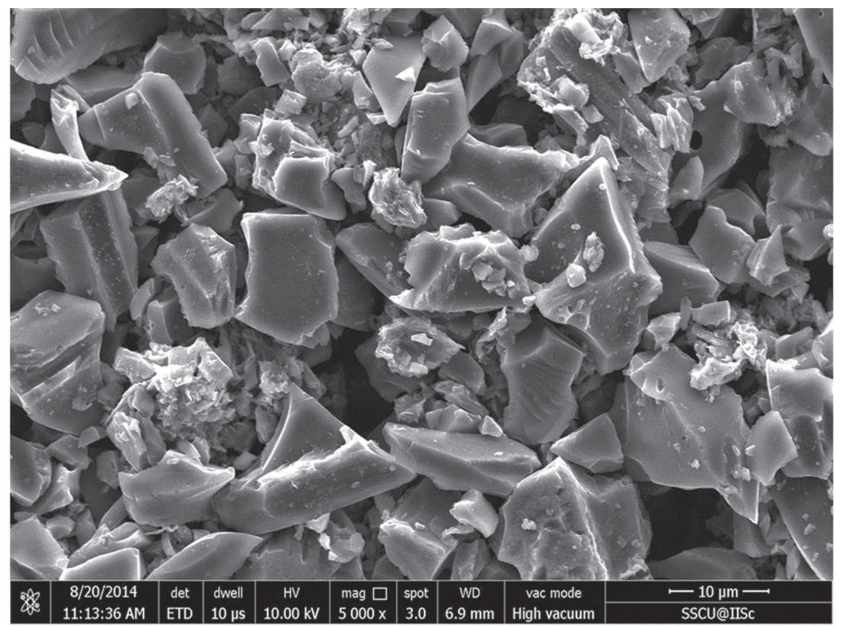

(a)

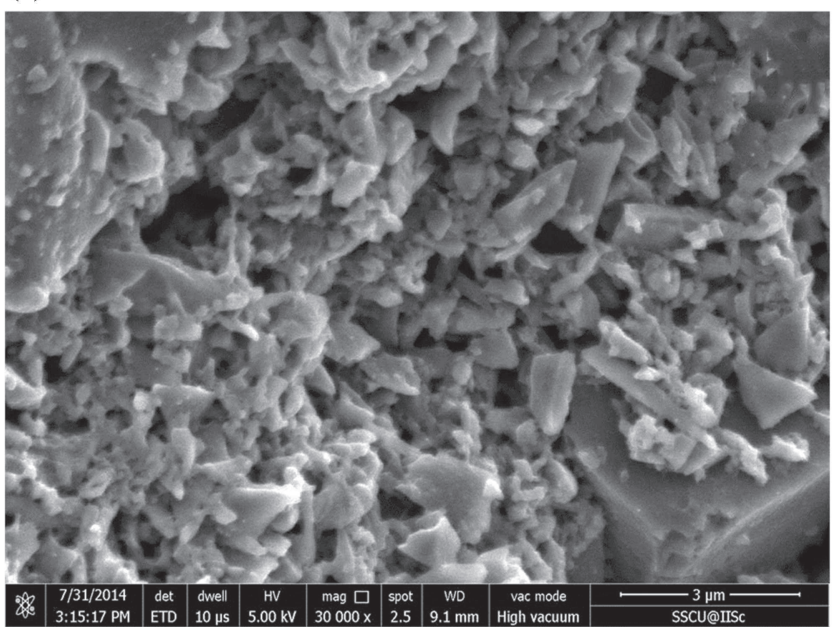

(b)

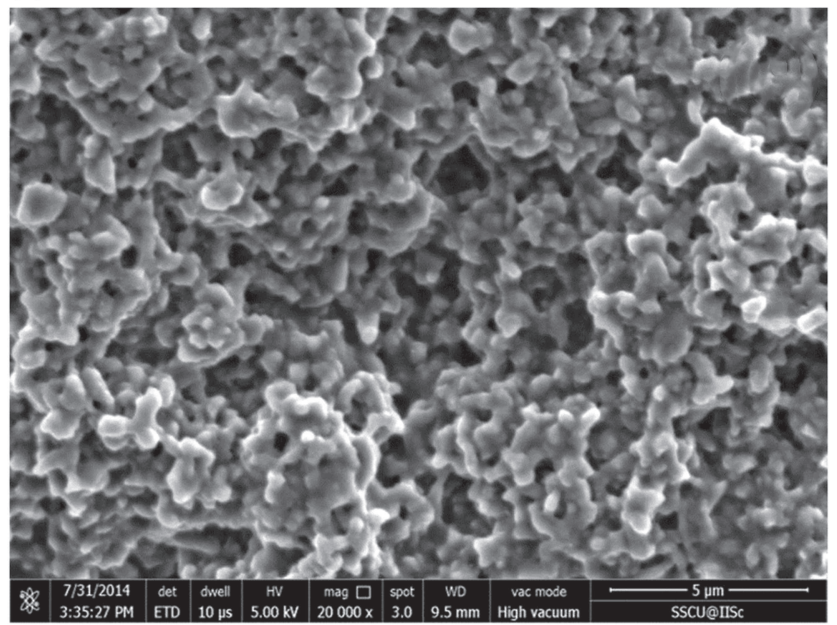

(c)

Figure 7. Scanning electron surface micrograph after 3000 cycling for (a) WE A, (b) WE B and (c) WE C. 

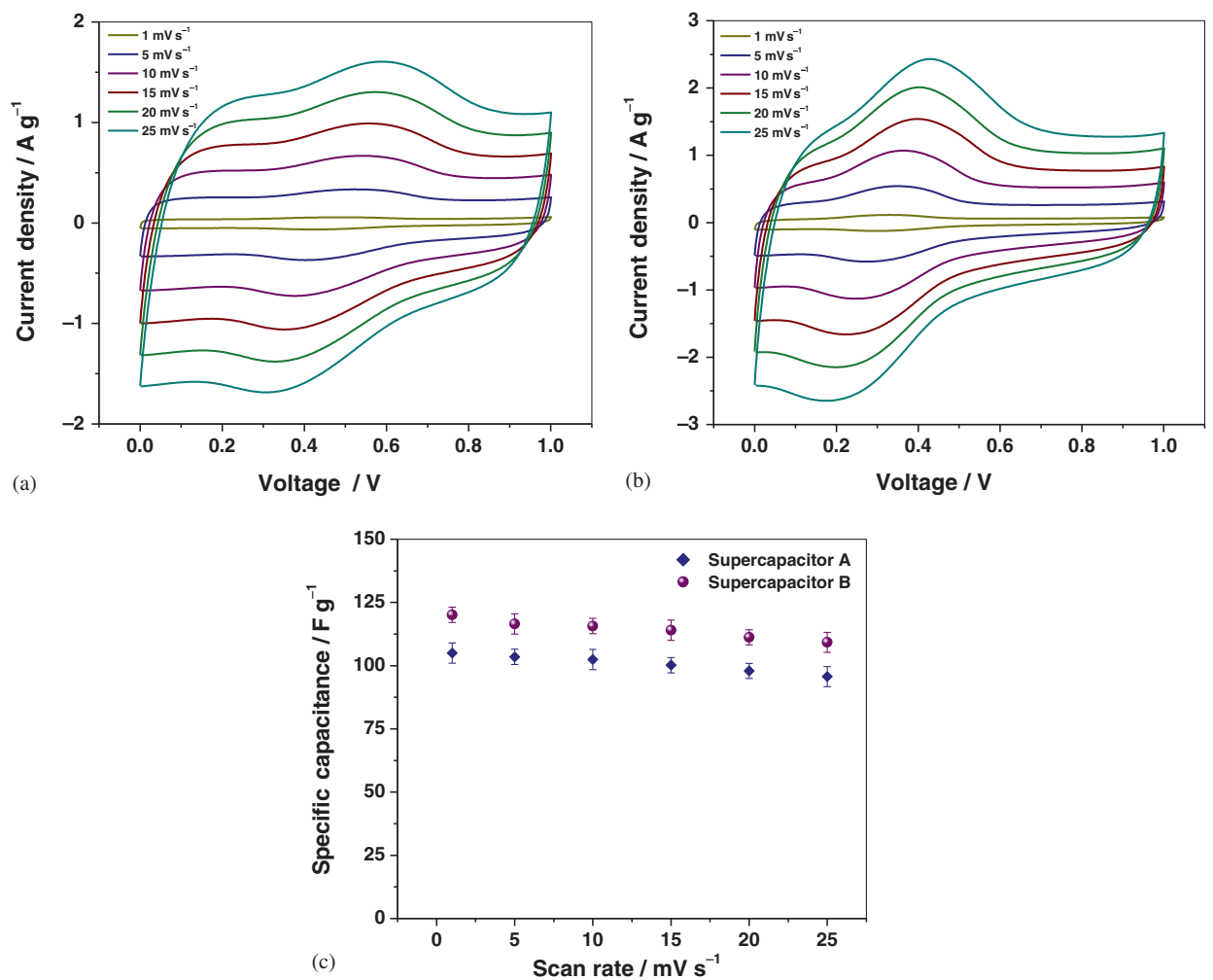

Figure 8. Cyclic voltammograms at different scan rates for (a) supercapacitor A and (b) supercapacitor B. (c) Scan rate vs. specific capacitance profiles for supercapacitors A and B.
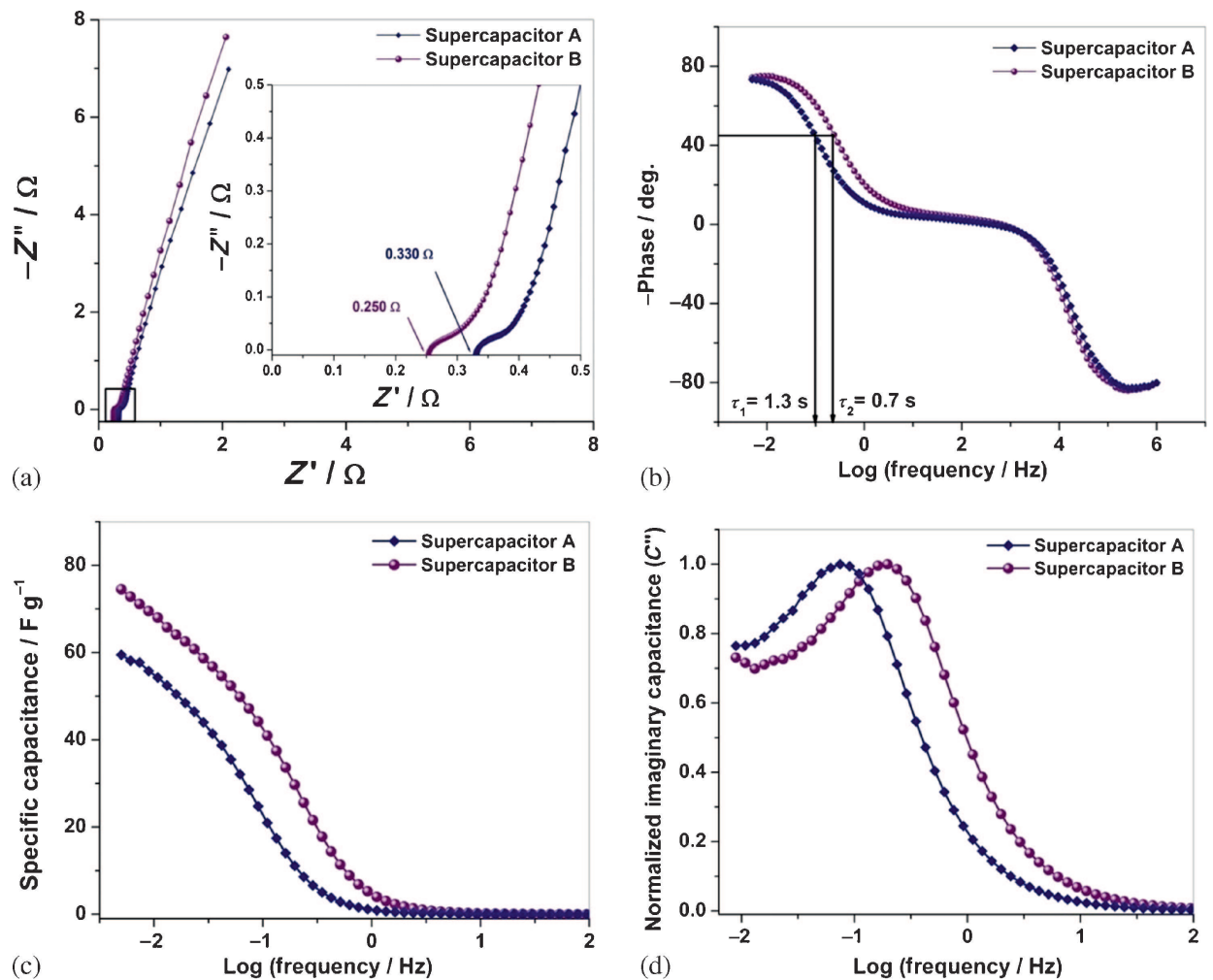

Figure 9. Frequency-response analysis by electrochemical impedance spectroscopy for supercapacitors A and B: (a) Nyquist plots, (b) Bode plots, (c) real capacitance $v s$. frequency plots and (d) imaginary capacitance $v s$. frequency plots. 
developed. The electrochemical performance tests, using cyclic voltammetry, ac impedance analysis, galvanostatic charge/discharge and cycle-life data, are carried out for $1 \mathrm{~V}$ supercapacitors as discussed below.

3.6a Cyclic voltammetry: Cyclic voltammograms for $1 \mathrm{~V}$ supercapacitors are shown in figure $8 \mathrm{a}$ and $\mathrm{b}$ at varying scan rates between 0 and $1 \mathrm{~V}$. Specific-capacitance values are calculated from cyclic voltammograms as reported in figure $8 \mathrm{c}$. Little deterioration in specific-capacitance values are observed at high scan rates.
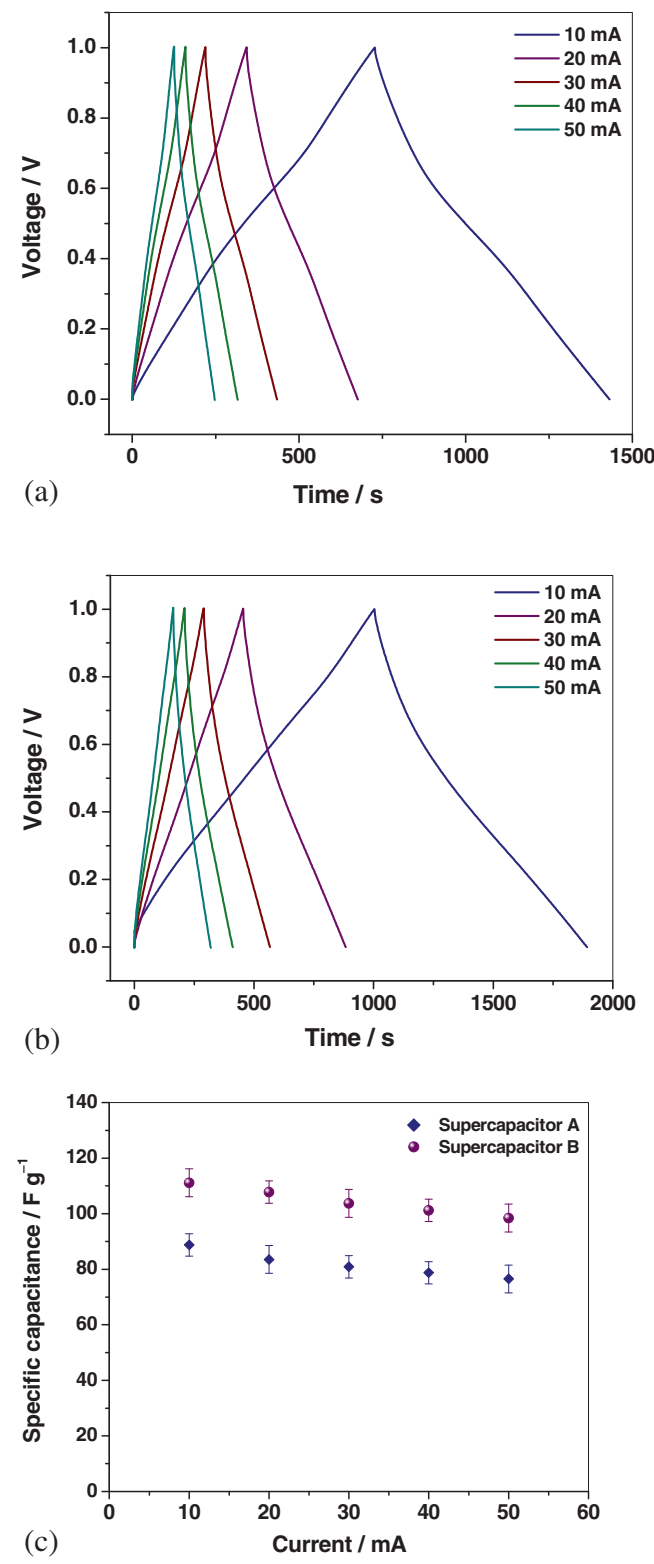

Figure 10. Galvanostatic charge/discharge profiles at different current densities for (a) supercapacitor A and (b) supercapacitor B. (c) Current density vs. specific capacitance profiles for supercapacitors $\mathrm{A}$ and $\mathrm{B}$. 3.6b Impedance analysis: Internal resistance and response-time values are estimated from Nyquist plots (figure 9a) and Bode plots (figure 9b), respectively. For supercapacitor $\mathrm{A}$, the internal resistance and response-time values are $0.33 \Omega$ and $1.3 \mathrm{~s}$, respectively, whereas for supercapacitor B, these respective values are $0.25 \Omega$ and $0.7 \mathrm{~s}$. The frequency response data for real and imaginary parts of capacitance are analysed, and respective profiles are shown in figure 9c and $\mathrm{d}$. These profiles correspond to single-electrode study and the response-time values from figure $9 \mathrm{~d}$ match with abovementioned data.

3.6c Galvanostatic charge/discharge data: Galvanostatic charge/discharge experiments are carried out between $10 \mathrm{~mA}$ $\left(120 \mathrm{~mA} \mathrm{~g}^{-1}\right)$ and $50 \mathrm{~mA}\left(600 \mathrm{~mA} \mathrm{~g}^{-1}\right)$ load currents within 0 to $1 \mathrm{~V}$ for supercapacitor $\mathrm{A}$ and supercapacitor $\mathrm{B}$, and respective profiles are shown in figure 10a and b. Specificcapacitance values are calculated at each load current, and presented in figure 10c, where small degradation is observed at higher current loads. From figure 10c, the rated specificcapacitance values for supercapacitor A and supercapacitor $\mathrm{B}$ are estimated as 80 and $100 \mathrm{~F} \mathrm{~g}^{-1}$, respectively, with more than $90 \%$ faradaic efficiencies.

3.6d Energy and power densities: Energy and power densities are calculated from rated specific-capacitance values $(C)$ using equations (7) and (8), respectively, ${ }^{28}$ where $V_{\max }$ and $V_{\min }$ are 1 and $0 \mathrm{~V}$, respectively.

$$
\begin{aligned}
& \text { Energy density }=\frac{C\left(V_{\max }^{2}-V_{\min }^{2}\right)}{2 \times m} \\
& \text { Power density }=\frac{\text { Energy density }}{\text { Response time }}
\end{aligned}
$$

Energy and power density values for supercapacitor A were estimated to be $11 \mathrm{Wh} \mathrm{kg}^{-1}$ and $30 \mathrm{~kW} \mathrm{~kg}^{-1}$, respectively, while the respective values for supercapacitor B were 14 Wh kg-1 and $72 \mathrm{~kW} \mathrm{~kg}^{-1}$. Higher power density value for supercapacitor B is attributed to the presence of GO template.

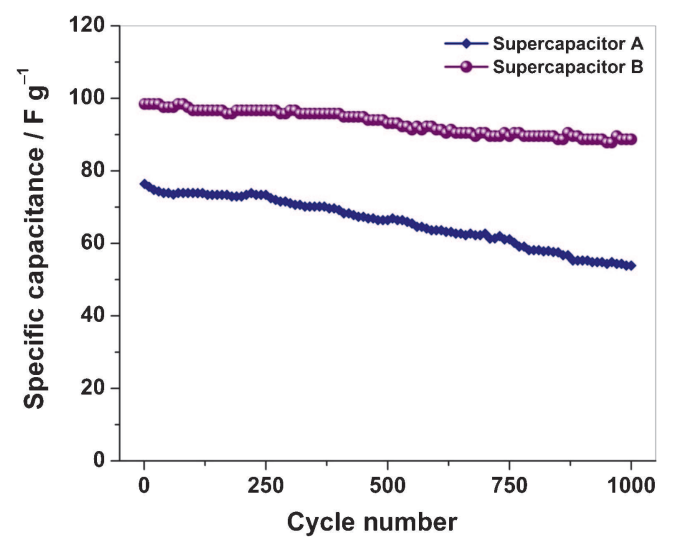

Figure 11. Cycle-life data for supercapacitors A and B at $50 \mathrm{~mA}$ current load. 


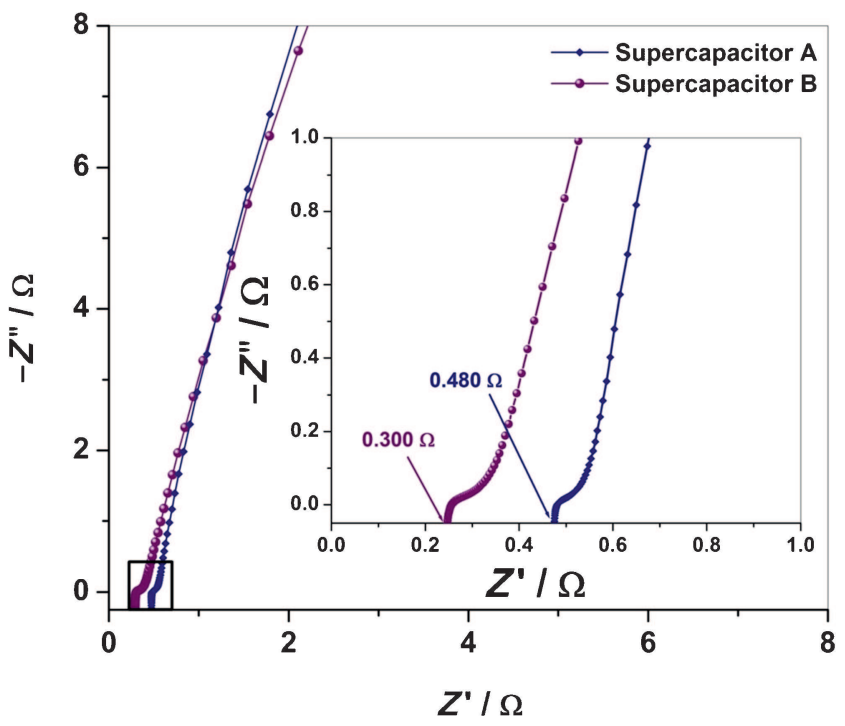

Figure 12. Nyquist plots with internal-resistance data after 1000 cycling for supercapacitors A and B.

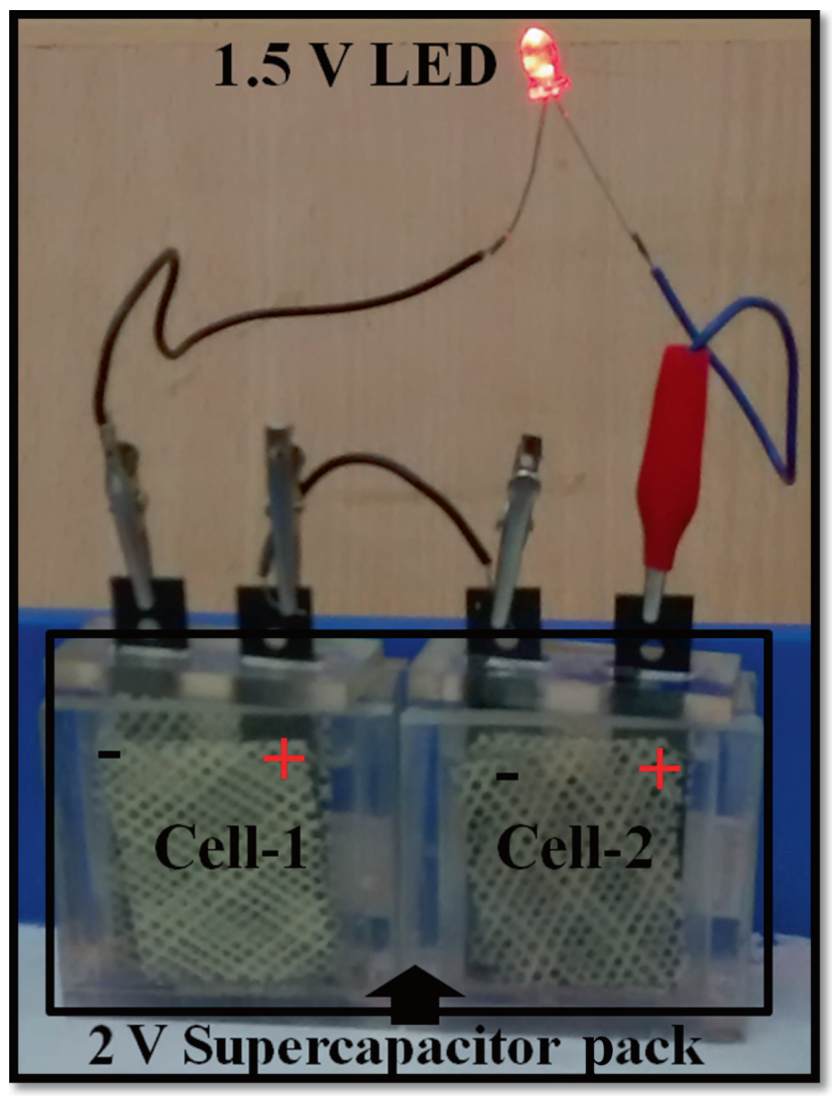

Figure 13. Photographic image of $2 \mathrm{~V}$ supercapacitor device pack under 1.5 V LED operation.

3.6e Cycle-life data: Cycle-life test was also performed at $50 \mathrm{~mA}\left(600 \mathrm{~mA} \mathrm{~g}^{-1}\right)$ load current for supercapacitor devices, and 1000 cycle data are presented in figure 11. It is seen that only 30 and $10 \%$ capacitance loss are observed after 1000 cycles for supercapacitors A and B, respectively.
Internal-resistance values are measured after 1000 cycles for supercapacitors $\mathrm{A}$ and $\mathrm{B}$, with respective values being 0.48 and $0.30 \Omega$ (figure 12). The increase in internal resistance is much higher for supercapacitor A in relation to supercapacitor B due to the breakdown of polymeric structure in supercapacitor A. It is noteworthy that the high cycling stability for supercapacitor B is owing to the retention of polymeric structures in GO/PANI.

3.6f Demonstration of $2 \mathrm{~V}$ supercapacitor pack with LED lamp: Figure 13 shows the design of $2 \mathrm{~V}$ supercapacitor pack with two $1 \mathrm{~V}$ cells in series under $1.5 \mathrm{~V}$ light emitting diode (LED) lamp operation. The real-life performance testing under 1.5 V LED load demonstrates the fabrication of functioning supercapacitor devices with nanostructured $\mathrm{GO}$ /polyaniline composite materials.

\section{Conclusions}

A GO/PANI composite-based supercapacitor device is reported, and a comparative study with PANI-based device is performed. In situ potentiostatic electropolymerization processes are followed for synthesizing PANI and GO/PANI composites, which are devoid of impurities, and contribute lesser internal resistance to the electrodes. Various electrochemical methods, namely cyclic voltammetry, impedance spectroscopy, galvanostatic charge/discharge and cycling stability, are used for characterization of polymer-based active materials in a three-electrode cell assembly and performance tests for $1 \mathrm{~V}$ supercapacitor devices. It is surmised that highpower capability and cycling stability make the GO/PANI composite-based supercapacitor a potential system for practical applications.

\section{Acknowledgements}

We are grateful to the Department of Science \& Technology, Government of India, for financial support. Deepak Kumar thanks University Grant Commission, New Delhi, for Dr D S Kothari Postdoctoral Fellowship (F.4-2/2006 (BSR)/13-899/2013).

\section{Electronic Supplementary Material}

Supplementary material pertaining to this article is available on the Bulletin of Materials Science website (www.ias.ac.in/ matersci).

\section{References}

1. Levi M D, Salitra G, Levy N, Aurbach D and Maier J 2009 Nat. Mater. 8872

2. Li H Q, Luo J Y, Zhou X F, Yu C Z and Xia Y Y 2007 J. Electrochem. Soc. 154 A731 
3. Jampani P, Manivannan A and Kumta P N 2010 Electrochem. Soc. Interface 1957

4. Wang Y and Xia Y 2006 J. Electrochem. Soc. 153 A450

5. Cong H P, Ren X C, Wang P and Yu S H 2013 Energy Environ. Sci. 61185

6. Peng X, Huo K, Fu J, Zhang X, Gao B and Chu P K 2013 Chem. Commun. 4910172

7. Hou Y, Cheng Y, Hobson T and Liu J 2010 Nano Lett. 10 2727

8. Zhou C, Zhang Y, Li Y and Liu J 2013 Nano Lett. 13 2078

9. Wang G, Zhang L and Zhang J 2012 Chem. Soc. Rev. 41797

10. Zhao Y, Liu B, Pan L and Yu G 2013 Energy Environ. Sci. 6 2856

11. Zhang Q, Li Y, Feng Y and Feng W 2013 Electrochim. Acta 9095

12. Du J and Cheng H-M 2012 Macromol. Chem. Phys. 2131060

13. Lerf A, He H, Forster M and Klinowski J 1998 J. Phys. Chem. B 1024477

14. Zhu Z Z, Wang G C, Sun M Q, Li X W and Li C Z 2011 Electrochim. Acta 561366

15. Zhang J and Zhao X S 2012 J. Phys. Chem. C 1165420

16. Fu H, Du Z-J, Zou W, Lia H-Q and Zhang C 2013 J. Mater. Chem. A 114943

17. Wang Y, Tao S, An Y, Wu S and Meng C 2013 J. Mater. Chem. A 18876
18. Liu T, Finn L, Yu M, Wang H, Zhai T, Lu X, Tong Y and Li Y 2014 Nano Lett. 142522

19. Kim J, Park S-J and Kim S 2013 Carbon Lett. 1451

20. Marcano D C, Kosynkin D V, Berlin J M, Sinitskii A, Sun Z, Slesarev A, Alemany L B, Lu W and Tour J M 2010 ACS Nano 44806

21. Stankovich S, Dikin D A, Piner R D, Kohlhaas K A, Kleinhammes A, Jia Y, Wu Y, Nguyen S T and Ruoff R S 2007 Carbon 451558

22. Li N, Xiao Y, Xu C, Li H and Yang X 2013 Int. J. Electrochem. Sci. 81181

23. Lu H, Liang F, Gou J, Leng J and Du S 2014 Smart Mater. Struct. 2385034

24. Ganguly A, Sharma S, Papakonstantinou P and Hamilton J 2011 J. Phys. Chem. C 11517009

25. Conway B E 1999 Electrochemical supercapacitors, scientific fundamentals and technological applications (New York: Kluwer Academic/Plenum Publishers).

26. Kumar N A, Choi H J, Shin Y R, Chang D W, Dai L and Baek J B 2012 ACS Nano 61715

27. Miller J R 1998 Proceedings of the 8th international seminar on double-layer capacitors and similar energy storage devices, Deerfield Beach, Florida, December 7-9 1998

28. Yang C, Li C Y V, Li F and Chan K Y 2013 J. Electrochem. Soc. $160 \mathrm{H} 271$

29. Srinivasan V and Weidner J W 1999 J. Electrochem. Soc. 146 1650 